\title{
11
}

\section{Optical Features of Catalyst-free Zinc Oxide Nanostructures Confined to one Dimension*}

\author{
(c) D. Soubane ${ }^{1,2}$, A. Tirbiyine ${ }^{3}$, M. Bellioua ${ }^{4}$, S. Laasri ${ }^{5}$ and A. Hajjaji ${ }^{5}$ \\ ${ }^{1}$ Advanced Laser Light Source, Centre Energie Matériaux Télécommunications, \\ Institut National de la Recherche Scientifique, Varennes, \\ Québec J3X 1S2 Canada \\ ${ }^{2}$ Mining and Materials Engineering, McGill University, Montreal, \\ Québec, H3A 2K6 Canada \\ ${ }^{3}$ Equipe des Matériaux Supraconducteurs, Université Ibn Zohr, Faculté des Sciences, \\ Agadir, Morocco \\ ${ }^{4}$ Laboratory of Condensed MatterPhysics and Nanomaterials for Renewable Energy, Université Ibn Zohr, \\ Agadir, Morocco \\ ${ }^{5}$ Laboratoire des sciences de l'ingénieur pour l'énergie, ENSAJ, Université Chouaib doukkali, \\ El Jadida, Morocco \\ e-mail: soubane@emt.inrs.ca, driss.soubane@alumni.unistra.fr
}

Received January 26, 2019

Revised January 26, 2019

Accepted March 15, 2019

We successfully fabricated polycrystalline zinc oxide catalyst-free nanorods by a successive ion layer adsorption and reaction process. We measured their optical transmittance, reflectance and thickness in addition to performing $\mathrm{X}$-ray diffraction and probing film microscopic topology. We extracted different optical constants such as absorption coefficient, band gap, complex refractive index and complex dielectric function and inferred the behavior of the optical conductivity and show the supremacy of the obtained $\mathrm{ZnO}$ nanostructure over a bulk material. It turns out that it is possible to obtain $\mathrm{ZnO}$ nanorods ultra-thin film with optical quality almost similar to those obtained by a contaminant metal catalyst approach requiring extra cost.

Key words: nanorods, catalyst-free, zinc oxide, optical constants, SILAR, quantum confinement, downscaling.

DOI: $10.21883 /$ OS.2019.09.48204.35-19

* Полная версия статьи опубликована в английской версии журнала. 